\title{
Polyacrylic Acid Functionalized Nanographene as a Nanocarrier for Loading and Controlled Release of Doxorubicin Hydrochloride
}

\author{
Yunping Chen, Yuanyuan Qi, and Bin Liu \\ School of Stomatology, Lanzhou University, Lanzhou 730000, China \\ Correspondence should be addressed to Bin Liu; liubkq@lzu.edu.cn
}

Received 11 June 2013; Accepted 4 August 2013

Academic Editor: Xingbin Yan

Copyright (c) 2013 Yunping Chen et al. This is an open access article distributed under the Creative Commons Attribution License, which permits unrestricted use, distribution, and reproduction in any medium, provided the original work is properly cited.

Graphene nanosheets (GNSs) were synthesized by direct current arc discharge and subsequently functionalized by highly hydrophilic polyacrylic acid (PAA). The loading and release of doxorubicin hydrochloride (DOX) on PAA-functionalized GNSs (PAA-GNSs) were investigated. PAA-GNSs were successfully prepared and verified by atomic force microscope (AFM), Fouriertransform infrared (FTIR) and ultraviolet-visible (UV-vis) spectroscopes, and thermogravimetric analysis (TGA). The PAA-GNSs contained about $10 \mathrm{wt} \%$ PAA which significantly improved the solubility of GNSs in aqueous media. The efficient loading of DOX onto the PAA-GNSs is as high as $2.404 \pm 0.167 \mathrm{mg} / \mathrm{mg}$ at the DOX concentration of $0.36 \mathrm{mg} / \mathrm{mL}$. Furthermore, the PPA-GNSs showed strong $\mathrm{pH}$-dependent behavior and efficient slow release ability. Therefore, PAA-GNSs are promising new materials for biological and medical applications.

\section{Introduction}

Recently, nanomaterial-based drug carriers have become a hot spot of research at the interface of nanotechnology and biomedicine [1-3]. The nanomaterial-based drug carriers include nanoparticles [4, 5], micelles [6], liposomes [7], nanofibers [8], and self-assembled polymer nanodevices [9]. An ideal drug carrier should be capable of controlled release (triggered release of drug under a characteristic environment of diseased cells) and drug protection until entering diseased cells, which aim to prevent early drug degradation and to increase the intracellular drug concentration. By using this system for drug delivery, we can reduce the side effects of drugs and enhance the therapeutic efficacy [10-14].

Graphene, a two-dimensional nanomaterial reported for the first time in 2004 [15], has emerged with many intriguing properties including electrical, thermal, optical, sensing [16, 17], high surface area, and biocompatibility [18]. The single or fewer layered structure of graphene provides richness for diversified surface chemistry on both sides of the sheet including edges [19]; therefore, graphene and its derivatives have been proposed to offer high therapeutic molecule loading capacity due to their high available surface area and have been explored as potential drug delivery systems [2023]. Since 2008, graphene oxide (GO) with different surface functionalization has been exploited as nanocarriers for loading of a variety of chemotherapy drugs including DOX [1, 24-27], camptothecin (CPT) [21, 28, 29], SN38 (an analog of CPT) [30], ellagic acid [31], and $\beta$-lapachone [32] by either physical adsorption or covalent conjugation. Liu et al. found that GO after PEGylation showed excellent physiological stability and could be utilized for the loading of waterinsoluble aromatic drug SN38 via $\pi-\pi$ stackings [30]. Yang et al. investigated the loading and release behaviors of DOX on GO sheets and found that the efficient loading of DOX on the GO sheets is as high as $2.35 \mathrm{mg} / \mathrm{mg}$ at the DOX concentration of $0.47 \mathrm{mg} / \mathrm{mL}$ [10]. Zhang et al. found that GO modified by sulfonic acid showed specific targeting ability to MCF-7 cells. Interestingly, two types of drug molecules, DOX and $\mathrm{CPT}$, which were loaded together on GO, realized synergistic effects of cancer cell killing in a dose-dependent manner [3]. However, from the previous reports, both GO and GNS were prepared by a modified Hummers method [33]. In this route, GO was generally prepared from graphite involving 
oxidation-exfoliation by using a mass of concentrated $\mathrm{H}_{2} \mathrm{SO}_{4}$ and $\mathrm{KMnO}_{4}$ strong oxidants which are not in favor of the environment protection. GO is not completely exfoliated and contains extensive domains of stacked graphitic layers [34]. In addition, unavoidable $\mathrm{MnO}_{2}$ impurity attached on GO sheets [35] would affect the subsequent reliability-safety of GO-based drug nanocarriers.

Arc discharge is a versatile and low-cost method for producing various kinds of carbon nanomaterials. Compared to other methods, the graphene sheets synthesized by arc discharge method have good dispersibility in organic solvent, few defects, and high thermal stability [35-39]. Recently, we have synthesized few-layered GNSs by direct current arc discharge using different buffer gases $[35,40]$. The GNSs have merits of high quality (no metallic impurity), excellent electrical conductivity, good thermal stability, and few defects. Additionally, the sizes of as-made GNSs are mainly in the range of 50-200 $\mathrm{nm}$ [35], which are much smaller than those of chemically reduced GO sheets. The relatively small GNSs would be suitable for biological applications, especially for drug nanocarriers. However, the GNSs are hydrophobic and tend to form agglomerates or even regraphitized to graphite due to the strong $\pi-\pi$ stacking and Van der Waals interactions which may limit their further biological applications [3, 41]. Therefore, the prevention for aggregation by functionalizing GNSs is of vital importance because most of its unique properties are only associated with individual sheets. In earlier report, chemically reduced GO sheets were functionalized by in situ polymerization of acrylic acid monomers, to generate PAA grafted graphene [42].

Herein, we have grafted acrylic acid monomers onto the GNSs surface by an in situ polymerization. The success of the functionalization is verified by AFM, FTIR and UV-vis spectroscopes, and TGA. The PAA-modified GNSs display good solubility in aqueous solutions and form stable dispersions for a long time. Due to the conjugated structure of graphene basal planes, PAA-GNSs can attach and absorb aromatic, water insoluble drugs [12]. And the amount of DOX loaded onto PAA-GNSs is significantly high and strongly dependent on $\mathrm{pH}$ value. Furthermore, the interaction between DOX and PAA-GNS was investigated by spectroscopy. It is believed that PAA-GNS could have great potential in biological applications for drug delivery.

\section{Experimental}

2.1. Materials. High-purity (99.99\%) graphite powder (325 mesh) was purchased from Qingdao Huatai Tech. Co., Ltd., China. N, N-dimethyl formamide (DMF) was purchased from Tianjin Reagent Company, China. Acrylic acid was purchased from Shanghai ShanPu Chemical Company, China. DOX was purchased from Shenzhen Main Luck Pharmaceuticals Inc., China. All chemicals and solvents were commercially available and were of analytical reagent grade. Dialysis tube (molecular weight cutoff: 8000-14000; diameter: $44 \mathrm{~mm}$ ) for the drug release was purchased from Beijing Solarbio Science \& Technology Co., Ltd., China.
2.2. Preparation of PAA-GNS. GNSs were prepared following our previous report [35]. Typically, a direct current arc discharge of the graphite was carried out in a water-cooled stainless steel chamber filled with $\mathrm{H}_{2}$ (200 Torr) and $\mathrm{He}$ (200 Torr). The cathode and anode were graphite rods (purity: 99.99\%) with diameters of 12 and $8 \mathrm{~mm}$, respectively. The current was kept at $140 \mathrm{~A}$ during the discharge process, and the arc was maintained by continuously adjusting the cathode to keep a constant distance of about $2 \mathrm{~mm}$ from the anode. After discharge, the graphene powders were collected.

PAA-GNSs was synthesized following the method reported [42]. GNSs $(20 \mathrm{mg})$ were added to a $250 \mathrm{~mL}$ dried three-necked flask, and $50 \mathrm{~mL}$ of solvent (1:9, DMF: $\mathrm{H}_{2} \mathrm{O}$ ) was loaded in. After ultrasonication for $30 \mathrm{~min}$ at $600 \mathrm{~W}$, the solution was purged under dry nitrogen for $60 \mathrm{~min}$ to remove the oxygen, followed by the addition of acrylic acid $(10 \mathrm{~g})$ and $40 \mathrm{~mL} \mathrm{H}_{2} \mathrm{O}$. Approximately $100 \mathrm{mg}$ of $\left(\mathrm{NH}_{4}\right)_{2} \mathrm{~S}_{2} \mathrm{O}_{8}$ (dissolved in $80 \mathrm{~mL} \mathrm{H}_{2} \mathrm{O}$ ) was then loaded in the flask using a dropping funnel. The flask was placed in an oil bath $\left(60^{\circ} \mathrm{C}\right)$ under stirring. After $48 \mathrm{~h}$, the mixture was cooled to room temperature, diluted with $200 \mathrm{~mL} \mathrm{H}_{2} \mathrm{O}$, and sonicated for $1 \mathrm{~h}$ then centrifuged. The GNS functionalized by PAA polymer were denoted as PAA-GNS.

\subsection{Loading of DOX and In Vitro Controlled Release Exper-} iments. The loading of DOX was performed by simple dissolving DOX in a PAA-GNS aqueous solution and stirred overnight. The free, unbound DOX was removed by centrifugation, repeated washing, and filtration. PAA-GNSs with the final concentration of $0.12 \mathrm{mg} / \mathrm{mL}$ (determined using a standard PAA-GNSs concentration curve at the absorption of $490 \mathrm{~nm}$ ) were first sonicated with DOX for $0.5 \mathrm{~h}$ and then stirred overnight at room temperature in the dark. All samples were adjusted to $\mathrm{pH}<6$ with $1 \mathrm{M}$ hydrochloride and then ultracentrifuged at $12000 \mathrm{rpm}$ for $1 \mathrm{~h} \mathrm{[10].}$

To determine the release rate of DOX loaded on PAAGNSs, the nanocarrier PAA-GNSs (6 mg) were dispersed in $3 \mathrm{~mL}$ of phosphate buffered saline (PBS), and the dispersion was divided into three equal aliquots. The PAA-GNSs samples used for the release experiments were placed into inner dialysis tubes, which were dialyzed in $50 \mathrm{~mL}$ of PBS buffer with $\mathrm{pH} 2,7$, and 10, respectively, in outside reservoir. The sealed reservoir was incubated in a shaker at $37^{\circ} \mathrm{C}$ for a period of 4 days. At selected time intervals, $2 \mathrm{~mL}$ dialyzate was taken out from the reservoir and equal volume of fresh PBS buffer was replenished to it. The released DOX was evaluated by measuring the UV-vis absorbance at $490 \mathrm{~nm}$.

2.4. Characterization. The morphology and structure analysis of the GNS was carried out on a high-resolution TEM (JEM-2010, at $200 \mathrm{kV}$ ). Raman spectrum was recorded by micro-Raman spectroscopy (JY-HR800, the excitation wavelength at $532 \mathrm{~nm}$ ), and AFM image was carried out by Nanoscope III, a multimode atomic force microscope (Veeco) in tapping mode, to investigate the structures and surface morphology of as-prepared GNS and PAA-GNS. The thermal property of the GNS, PAA, and PAA-GNS samples 


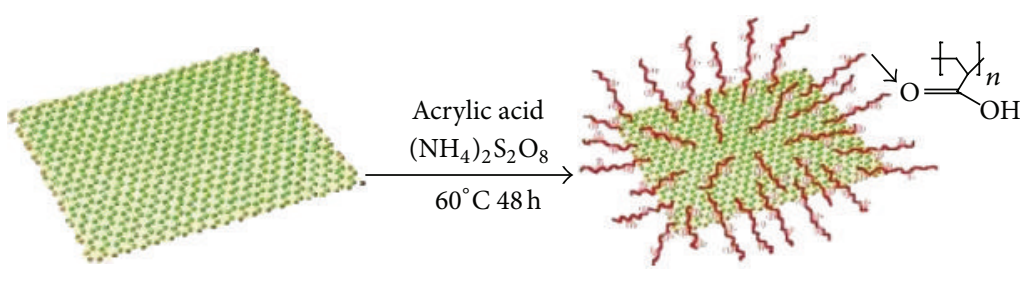

(a)

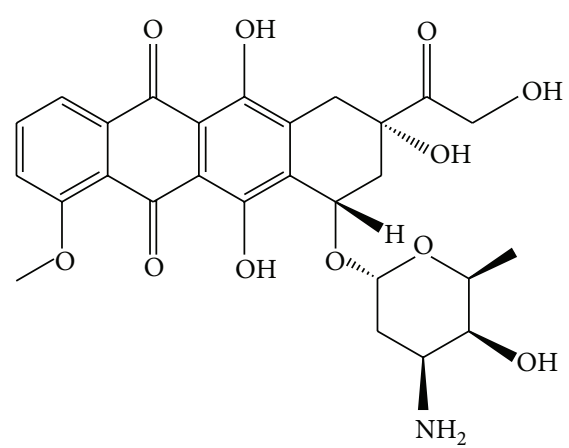

(b)

Scheme 1: (a) Schematic illustration of the generation of PAA-GNS nanocarrier, and (b) the structure of DOX molecule.

was conducted through a thermogravimetric analyzer (TGADSA 2960, TA Instruments) carried out from RT to $1000^{\circ} \mathrm{C}$ at $5^{\circ} \mathrm{C}$ min $^{-1}$ under $\mathrm{N}_{2}$ atmosphere. The microstructure of GNS, PAA-GNS, DOX, DOX-GNS, and DOX-PAA-GNS was characterized by Fourier transform infrared spectrometer (Bruker IFS66V). The loading capacity of DOX by the GNS and PAA-GNS were measured by a UV-vis spectrophotometer (Specord50, analytic jena, Germany) at $490 \mathrm{~nm}$.

\section{Results and Discussion}

Figure 1(a) shows the TEM image of the graphene sheets, from which transparent graphene sheets are present over a large area, indicating that the graphene sheets have few layers and high purity. Also, the surfaces of the graphene sheets are not perfectly flat, with wrinkles over them giving different levels of transparency. The geometric means of the length and width of each sheet were used to define their size, which was in the range 50-100 nm. Figure 1(b) shows a typical Raman spectrum of the GNS powders. Three intense peaks are the D band at $1345 \mathrm{~cm}^{-1}$, the $\mathrm{G}$ band at $1580 \mathrm{~cm}^{-1}$, and the $2 \mathrm{D}$ band at $2695 \mathrm{~cm}^{-1}$, respectively. The $D$ peak located at $1345 \mathrm{~cm}^{-1}$ is due to the presence of structural disorders in graphene sheets. The $G$ peak corresponds to the stretching vibration mode, $E_{2 \mathrm{~g}}$ phonon at the Brillouin zone center. The (2D) peak is always present for crystalline carbon materials [35].

There are bare of groups on the GNS sheets produced by arc discharge method. To realize good physiological stability and form stable dispersions, we develop a simple and convenient route to prepare functional GNS. Here, hydrophilic and biocompatible PAA are assembled onto GNS sheets to improve the aqueous solubility of the graphene. Scheme 1 shows the synthetic route for the preparation of PAA-GNS conjugate. The large $\pi$ conjugated structure of GNS can form $\pi-\pi$ stacking interaction with the quinone portion of DOX. Amino $\left(-\mathrm{NH}_{2}\right)$ and several hydroxyl $(-\mathrm{OH})$ groups are also on DOX. The carboxylic $(-\mathrm{COOH})$ groups on the PAAGNS sheet can form a strong hydrogen-bonding interaction with $-\mathrm{OH}$ and $-\mathrm{NH}_{2}$ groups of DOX. Therefore, DOX is noncovalently loaded on PAA-GNS simply by mixing them in aqueous solution with the aid of slight sonication [10].
Successful functionalization of GNS is first evidenced by the high solubility and stability of PAA-GNS in salt and cellular solutions. After removing the residual AA by washing with distilled water and centrifugation, the precipitated PAAGNS is found to be well dispersed in distilled water, PBS, and cell culture, respectively. It is stable after storage for $24 \mathrm{~h}$, showing high stability (Figure 2). PEGylation of graphene derivatives was usually used to improve the stability of graphene derivatives under physiological conditions [15]. Instead of using expensive and large PEG molecules, we render the GNS stable in physiological solution by simple in situ polymerization with PAA that would graft many $-\mathrm{COOH}$ groups on the surface of GNS.

The morphology of GNS before and after functionalization is characterized with AFM in tapping mode, as shown in Figure 3. GNS is small sheets ranging from 50 to $100 \mathrm{~nm}$ and shows smooth and flat surface (Figure 3(a)). In contrast, the thickness of PAA-GNS is increased and many surface protuberances are observed on the surface of PAA-GNS and the sheet edges turn to be coarse, as shown in Figure 3(b). Obviously, PAA chains are wrapped onto the GNS sheet.

From the FTIR spectra of GNS and PAA-GNS in Figure 4(a), there are no groups on the GNS sheets. After functionalization, we can see the characteristic absorbance bands of PAA. The peak of $\mathrm{C}=\mathrm{O}$ vibration suggests that the PAA is firmly introduced into GNS. The $-\mathrm{COOH}$ groups at the PAA terminals are available for bioconjugation. From the UV-vis absorption spectra (Figure 4(b)), the absorption peak of GNS is at $270 \mathrm{~nm}$, which is consisted with the reported result [3], while the PAA-GNS shows a characteristic absorption peak of PAA at $216 \mathrm{~nm}$. Both FTIR and UV-vis spectra confirm the success of functionalization.

TGA is a complementary technique that can reveal the composition and changes in thermal stability of the PAAGNS. From Figure 5, it is observed that GNS produced by arc discharge method exhibits a high thermal stability. There is hardly any weight loss under $300^{\circ} \mathrm{C}$ and a small weight loss of $\sim 2 \%$ in the range $350-450^{\circ} \mathrm{C}$. Compared to GNS, PAA is thermally unstable and starts to lose mass upon heating even below $100^{\circ} \mathrm{C}$, which is attributed to the volatilization of residuary water in its structure. The major mass loss occurs in the range $230-450^{\circ} \mathrm{C}$, which is attributed to the pyrolysis of labile oxygen-containing groups. For PAA-GNS, the curve 


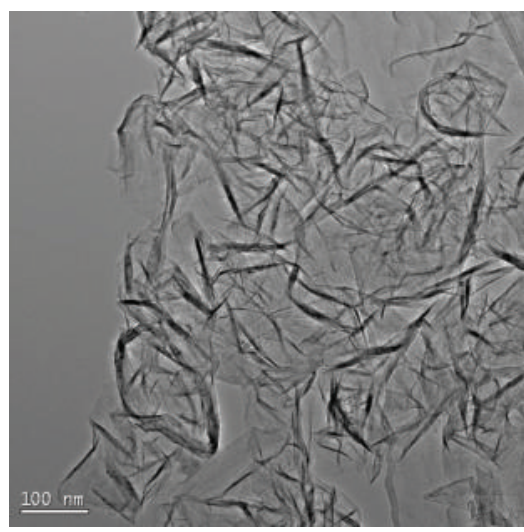

(a)

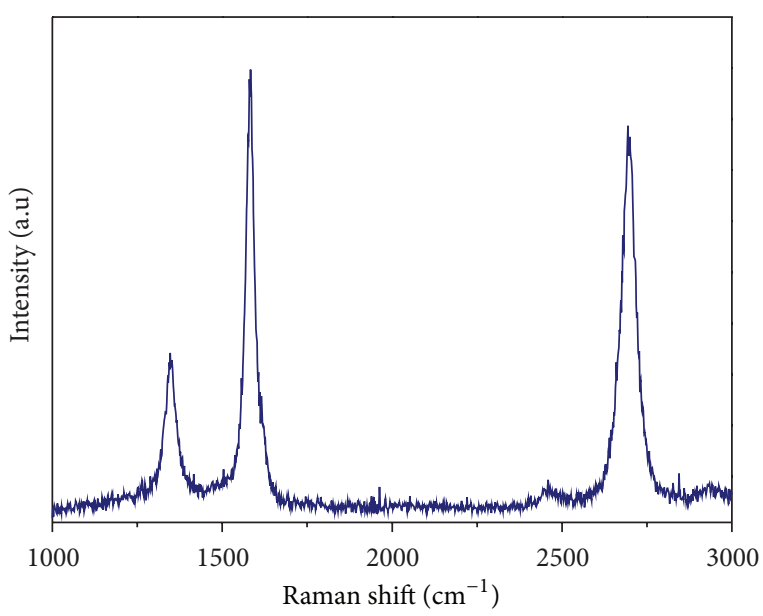

(b)

FIGURE 1: TEM images (a) and Raman spectrum (b) of GNSs produced by arc discharge in $\mathrm{H}_{2}$-He.

shows two-step thermal decomposition behavior. Between 150 and $300^{\circ} \mathrm{C}$, the curves show the first decomposition from the PAA-GNS, indicating the successful polymer attachment to the surface of the GNSs. The second decomposition occurs when the GNS sheets began to decompose from $300^{\circ} \mathrm{C}$ until the end. Thus, clearly, the decomposition profile of PAA-GNS is different from that of GNS and PAA. The weight reductions can be calculated for the PAA-GNS. The mass fraction of PAA on the GNS is about $10 \%$.

The evidence of DOX loading on PAA-GNS comes from the UV-vis spectra (Figure 6). The absorption peaks of the free DOX are shown at 233, 253, 291, and $490 \mathrm{~nm}$. The stacking of DOX onto PAA-GNS is evident from the spectrum of the GO-DOX nanohybrid solution, which shows the characteristic absorption peaks of DOX clearly. As the absorption peaks of DOX are so strong, the peaks of PAAGNS are not obvious (Figure 4(b)).

The loading capacity of DOX on PAA-GNS is determined by UV-vis spectrum at $490 \mathrm{~nm}$, which is calculated by the difference of DOX concentrations between the original DOX solution and the supernatant solution after loading. On the basis of standard curve of DOX absorbance
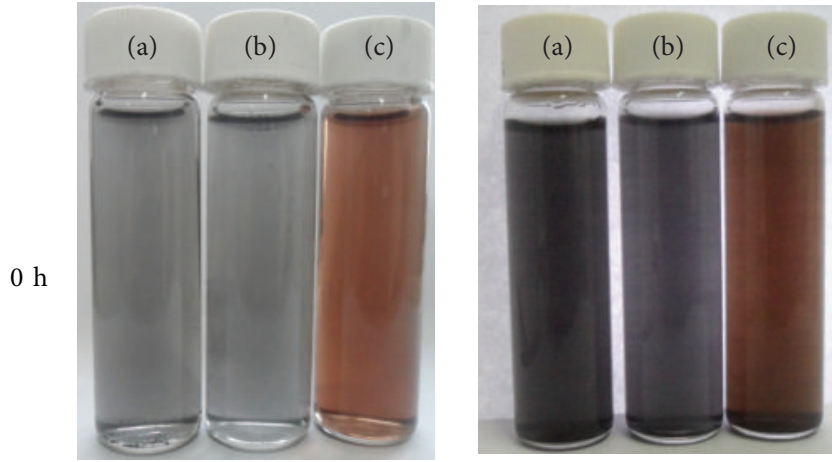

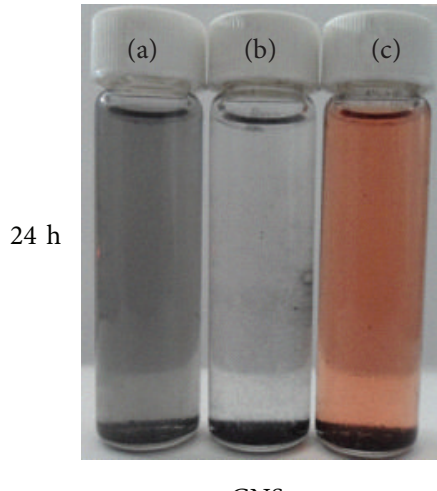

GNS

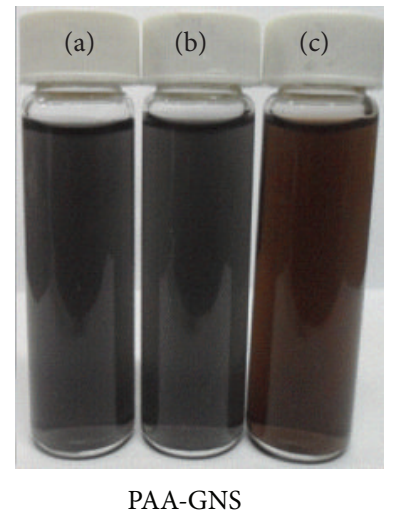

FIGURE 2: Photographs of GNS and PAA-GNS dispersed in water (a), PBS (b), and cell medium (c), stored for $0 \mathrm{~h}$ and $24 \mathrm{~h}$, respectively.

to its concentration, we can calculate the amount of DOX loaded on PAA-GNS which is determined using the following equation:

$$
\Phi=\frac{\left(M_{\mathrm{DOX}}-M_{\mathrm{DOX}^{\prime}}\right)}{M_{\mathrm{PAA}-\mathrm{GNS}}},
$$

where $\Phi$ is the amount of DOX loaded on PAA-GNS, $M_{\mathrm{DOX}}$ is the initial amount of DOX, $M_{\mathrm{DOX}^{\prime}}$ is the amount of DOX in the upper layer after centrifugation, and $M_{\text {PAA-GNS }}$ is the added amount of PAA-GNS.

The loading of DOX on PAA-GNS is investigated in different initial DOX concentrations with respect to the same concentration of PAA-GNS $(0.12 \mathrm{mg} / \mathrm{mL})$, as shown in Figure 7, and the loading ratio is correlated to the concentration of drug. The loading of DOX on PAA-GNS is $0.311 \pm$ $0.060 \mathrm{mg} / \mathrm{mg}$ when the DOX concentration is $0.06 \mathrm{mg} / \mathrm{mL}$. With the increase of the initial DOX concentration, the loading capacity of DOX increases and reaches $2.404 \pm$ $0.167 \mathrm{mg} / \mathrm{mg}$ when the DOX concentration is $0.36 \mathrm{mg} / \mathrm{mL}$, which can be seen as the saturated loading efficiency of PAA-GNS toward DOX. With further increase of DOX concentration, the loading capacity would not increase. It is related with the chemical structure of DOX. The interaction between PAA-GNS and DOX may come from $\pi-\pi$ staking between the conjugated structure of GNS sheet and the quinone portion of DOX and the hydrophobic effect between them. Also, the hydrogen bonding between the $-\mathrm{COOH}$ 


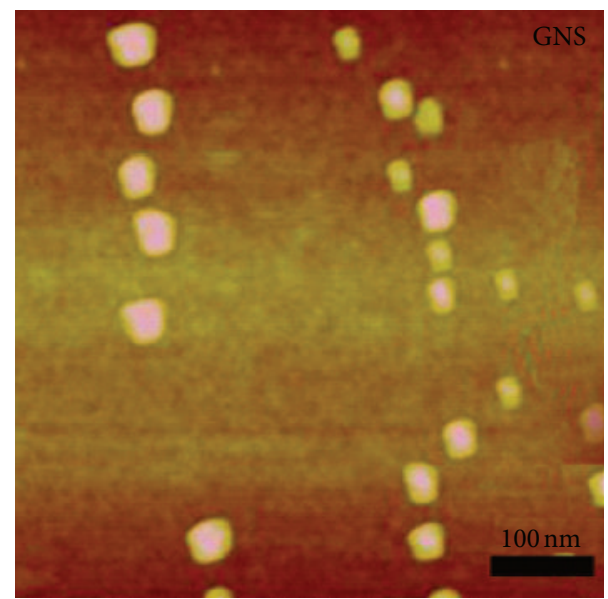

(a)
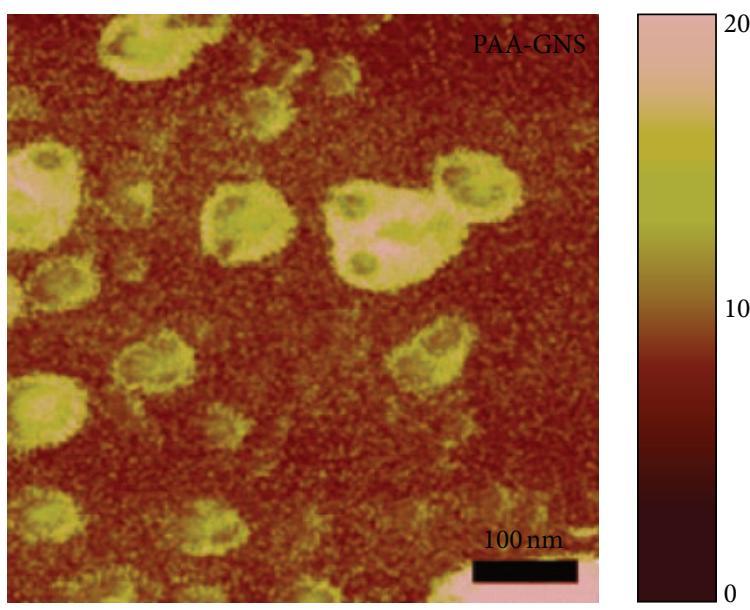

(b)

FIGURE 3: AFM images of GNS (a) and PAA-GNS (b).

groups of PAA-GNS and the $-\mathrm{OH}$ and $-\mathrm{NH}_{2}$ groups of $\mathrm{DOX}$ is existed.

Figure 8 shows the loading of DOX on GNS-PAA at the initial DOX concentration of $0.36 \mathrm{mg} / \mathrm{mL}$ with $\mathrm{pH}$ values of 2,7 , and 10 . The loading of DOX is $1.434 \mathrm{mg} / \mathrm{mg}$ at $\mathrm{pH}$ $2,2.504 \mathrm{mg} / \mathrm{mg}$ at $\mathrm{pH} 7$, and $1.801 \mathrm{mg} / \mathrm{mg}$ at $\mathrm{pH} 10$. It is observed that the loading capacity in the neutral condition is much higher than in acidic or basic conditions. This $\mathrm{pH}$ dependent behavior may be due to the different degree of hydrogen-bonding interaction between DOX and GNS-PAA under different $\mathrm{pH}$ conditions. At $\mathrm{pH} 7$, there are two kinds of hydrogen bonding forming between - $\mathrm{COOH}$ of GNSPAA and $-\mathrm{NH}_{2}$ of DOX and - $\mathrm{COOH}$ of GNS-PAA and $\mathrm{OH}$ of DOX. Under acidic condition, the $\mathrm{H}^{+}$will compete with the hydrogen-bond-forming groups and then weaken the previous hydrogen-bonding interaction. However, at $\mathrm{pH}$ 10 , most of the $-\mathrm{COOH}$ of GNS-PAA changed into $-\mathrm{COO}^{-}$ and could not form or weaken the hydrogen bonding forming between $-\mathrm{COOH}$ of GNS-PAA and the $-\mathrm{NH}_{2}$ or $-\mathrm{OH}$ of DOX $[3,10]$.

Except the previous hydrogen-bonding interaction, the $\pi-\pi$ staking between the conjugated structure of GNS sheet

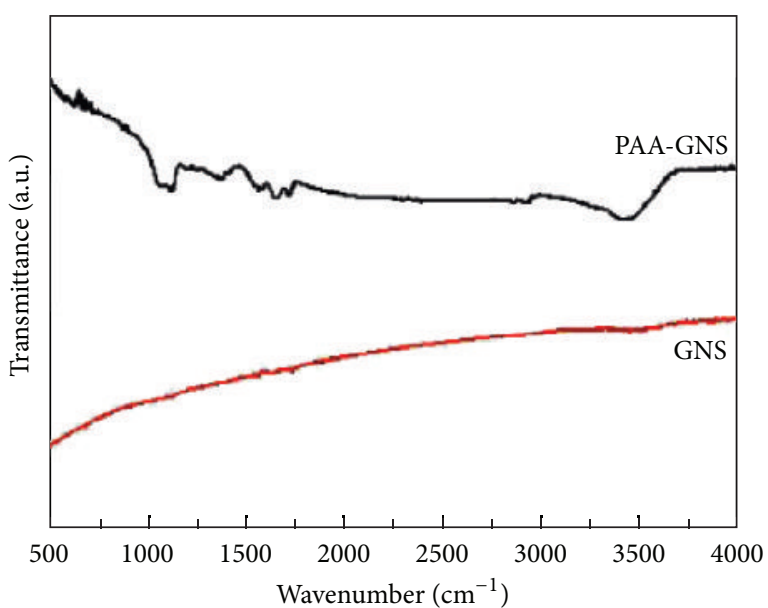

(a)

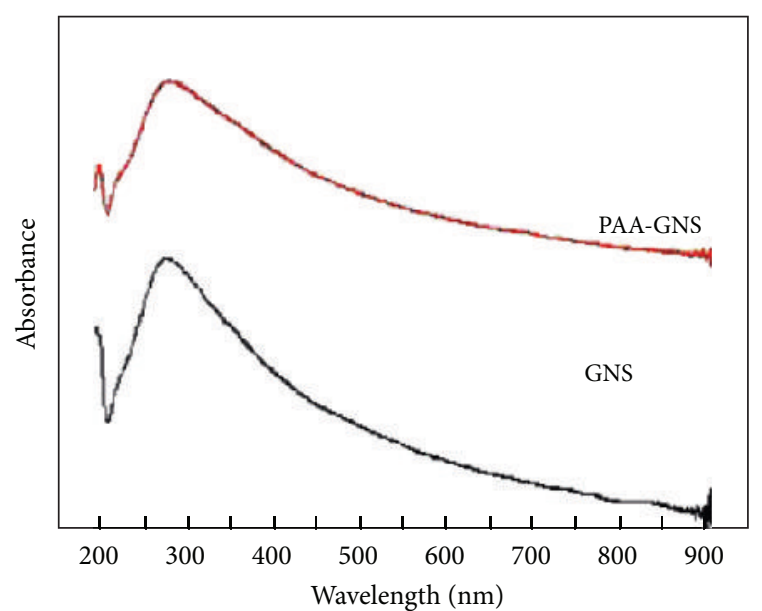

(b)

FIGURE 4: FTIR spectra (a) and UV absorption spectra (b) of GNS and PAA-GNS.

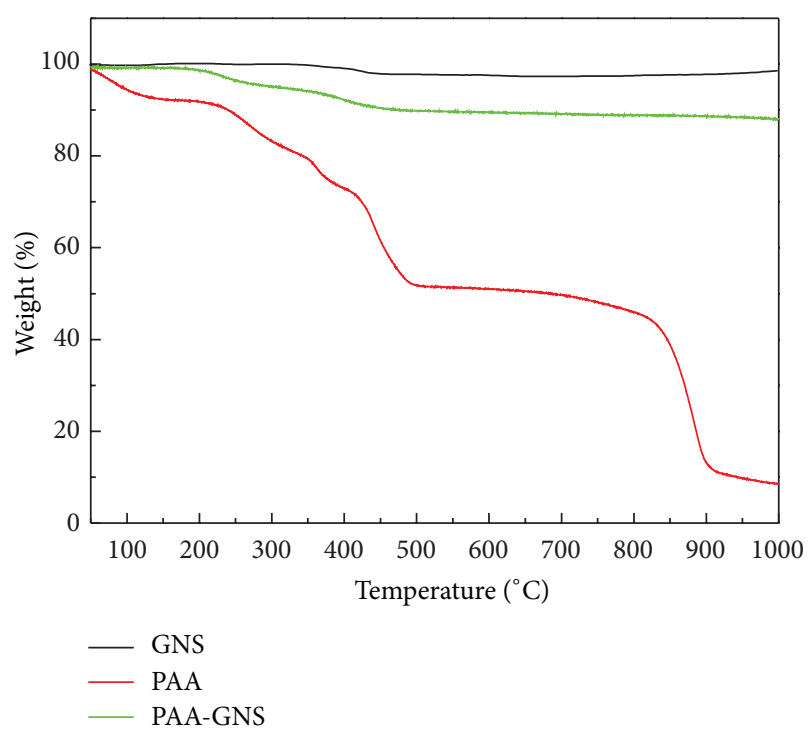

FIGURE 5: TGA curves of GNS, PAA, and PAA-GNS. 


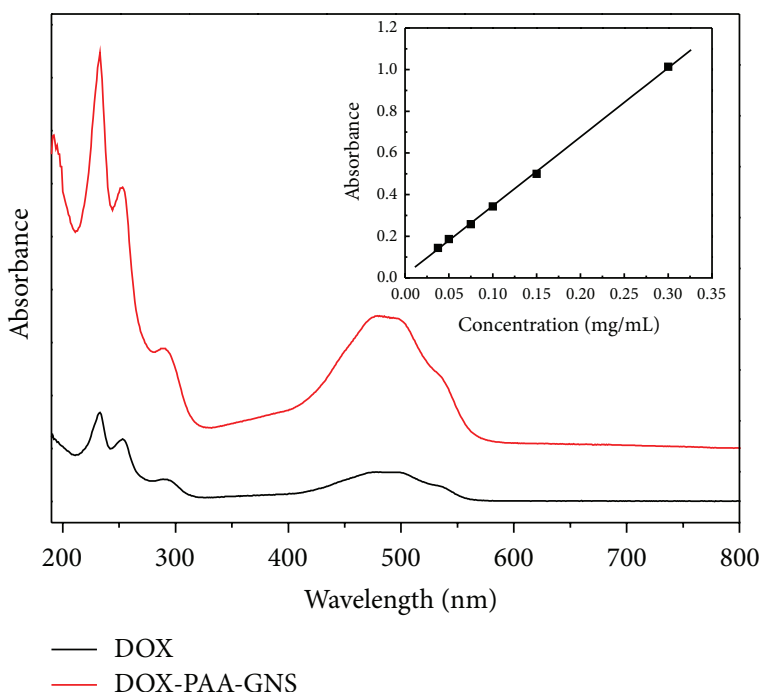

FIGURE 6: UV-vis absorption of DOX and DOX-PAA-GNS in aqueous solutions. The DOX absorbance at $490 \mathrm{~nm}$ was used to determine the loading efficiency (inset is the standard curve of DOX absorbance to its concentration).

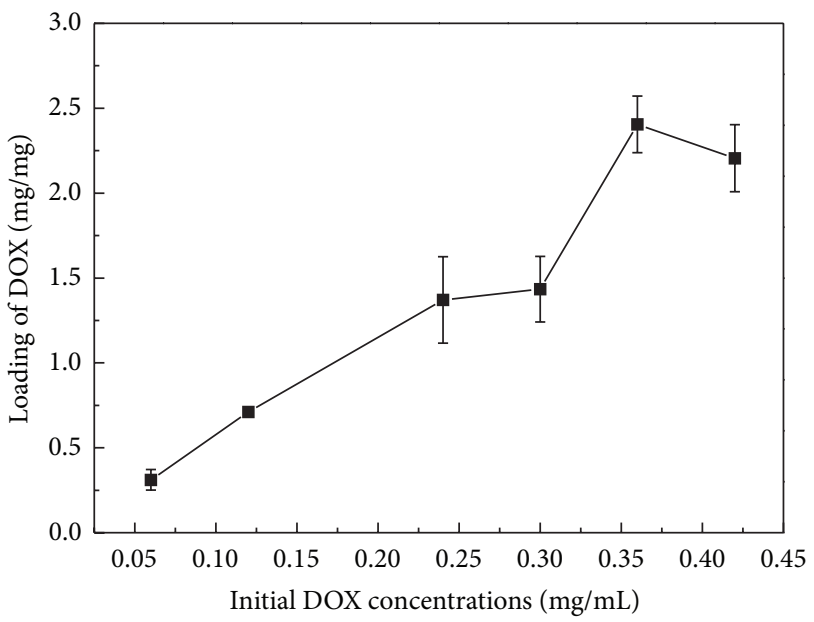

FIGURE 7: The loading capacity of DOX on PAA-GNS in different initial DOX concentrations.

and the quinone portion of DOX may be the most important interaction because the loading of DOX on GNS-PAA is still much high with the decreasing of the hydrogen bonding at pH 2. From the Figure 9, DOX exhibits a fluorescence emission maximum at $566 \mathrm{~nm}$ with an excited source at $480 \mathrm{~nm}$. However, GNS-PAA-DOX exhibited significant quenching of its emission band at the same wavelength. It implies the presence of a photoinduced electron-transfer process or efficient energy transferring between the GNS-PAA and DOX interface. According to the earlier report, this efficient quenching of fluorescence emission shows that there is strong $\pi-\pi$ stacking interaction between GNS-PAA and DOX [10, 43].

The release behavior of DOX from PAA-GNS is shown in Figure 10. At different $\mathrm{pH}$ environment, the release behavior

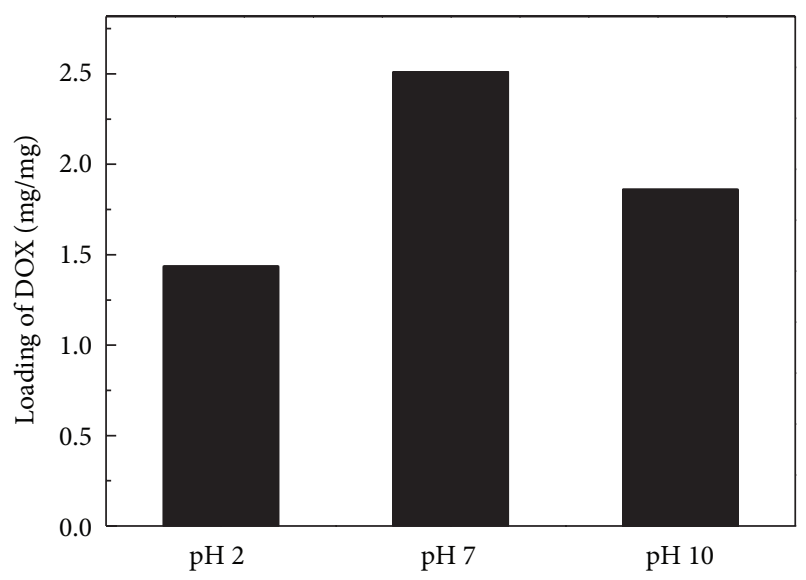

FIGURE 8: The loading capacity of DOX on PAA-GNS at different $\mathrm{pH}$ values.

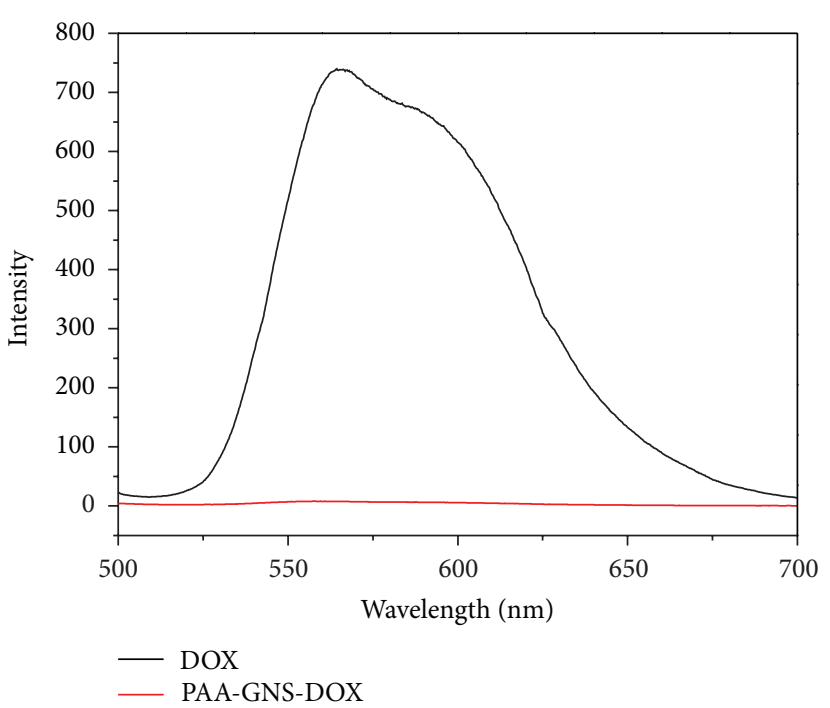

FIGURE 9: Fluorescence spectra of PAA-GNS-DOX and DOX in water at the $480 \mathrm{~nm}$ excitation wavelength. The concentrations of both PAA-GNS-DOX and DOX were all controlled to be the same according to the loading of DOX on PAA-GNS.

varies. It is found that $68 \%$ of DOX loaded on PAA-GNS is released after $48 \mathrm{~h}$ at $\mathrm{pH} 2$, while much less DOX is released after $48 \mathrm{~h}$ at $\mathrm{pH}$ 7.0. As discussed previously, the hydrogen-bonding interaction between DOX and GNS-PAA is the strongest at $\mathrm{pH} 7$, resulting in an inefficient release. Moreover, at low $\mathrm{pH}, \mathrm{DOX}$ becomes more hydrophilic and water soluble; thus, it leads to the release of more DOX from the PAA-GNS into PBS solution and the partial dissociation of hydrogen bonding interaction under acid condition. In addition, the DOX molecule has an $-\mathrm{NH}_{2}$ group, so its hydrophilicity becomes higher under acidic conditions, which explains why the release ratio of DOX in $\mathrm{pH} 2$ was about twice that of in $\mathrm{pH} 7$. However, DOX would become extremely volatile and decompose easily under alkaline conditions when meeting the light $[44,45]$, which may be the reason why the release ratio of $\mathrm{DOX}$ in $\mathrm{pH} 2$ is about seven 


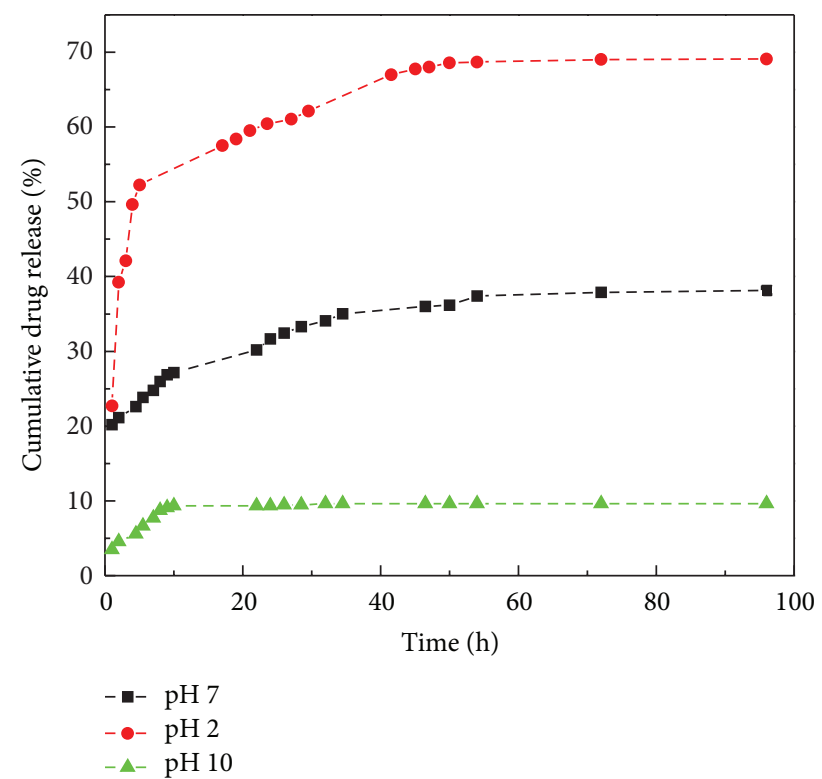

Figure 10: In vitro release profiles of DOX from PAA-GNS in PBS buffers with different $\mathrm{pH}$ values.

times of in $\mathrm{pH}$ 10. The $\mathrm{pH}$-dependent drug release from PAA-GNS could be exploited for drug delivery applications since the micro-environments in the extracellular tissues of tumors and intracellular lysosomes and endosomes are acidic, which will afford active drug release from PAA-GNS delivery vehicles.

\section{Conclusions}

In this paper, we have reported the synthesis of a functional GNS (PAA-GNS) with high aqueous solubility and stability in physiological solutions and studied its application on the loading and controlled release of a cancer drug. A high loading capacity and $\mathrm{pH}$-dependent release of DOX on PAAGNS are found. The loading of DOX on PAA-GNS increases with the increasing of initial DOX concentration, $2.404 \pm$ $0.167 \mathrm{mg} / \mathrm{mg}$ at the initial DOX concentration of $0.36 \mathrm{mg} / \mathrm{mL}$, and the highest release amount of the DOX from PAAGNS shows at $\mathrm{pH} 2$ then $\mathrm{pH} 7$ and $\mathrm{pH} 10$. Altogether, the water soluble PAA-GNS nanocarrier could open up a window to potential use of DOX delivery, which may have potential clinical advantages pertaining to increased therapeutic efficacy. The unique 2D shape and small size of PAA-GNS may offer interesting in vitro and in vivo behaviors.

\section{Disclosure}

The authors declare that they have no financial and personal relationships with other people or organizations that can inappropriately influence their works; there is no professional or other personal interest of any nature or kind in any product, service, and/or company that could be construed as influencing the position presented in, or the review of, the paper entitled.

\section{References}

[1] D. Depan, J. Shah, and R. D. K. Misra, "Controlled release of drug from folate-decorated and graphene mediated drug delivery system: synthesis, loading efficiency, and drug release response," Materials Science and Engineering C, vol. 31, no. 7, pp. 1305-1312, 2011.

[2] M. Ferrari, "Cancer nanotechnology: opportunities and challenges," Nature Reviews Cancer, vol. 5, no. 3, pp. 161-171, 2005.

[3] L. M. Zhang, J. G. Xia, Q. H. Zhao, L. W. Liu, and Z. J. Zhang, "Functional graphene oxide as a nanocarrier for controlled loading and targeted delivery of mixed anticancer drugs," Small, vol. 6, no. 4, pp. 537-544, 2010.

[4] S. B. Seo, J. Yang, W. Hyung et al., "Novel multifunctional PHDCA/PEI nano-drug carriers for simultaneous magnetically targeted cancer therapy and diagnosis via magnetic resonance imaging," Nanotechnology, vol. 18, no. 47, Article ID 475105, pp. 5105-5113, 2007.

[5] R. Singh and J. W. Lillard Jr., "Nanoparticle-based targeted drug delivery," Experimental and Molecular Pathology, vol. 86, no. 3, pp. 215-223, 2009.

[6] H. T. Ta, C. R. Dass, and D. E. Dunstan, "Injectable chitosan hydrogels for localised cancer therapy," Journal of Controlled Release, vol. 126, no. 3, pp. 205-216, 2008.

[7] M. A. Tran, R. J. Watts, and G. P. Robertson, "Use of liposomes as drug delivery vehicles for treatment of melanoma," Pigment Cell and Melanoma Research, vol. 22, no. 4, pp. 388-399, 2009.

[8] E. R. Kenawy, F. I. Abdel-Hay, M. H. El-Newehy, and G. E. Wnek, "Controlled release of ketoprofen from electrospun poly(vinyl alcohol) nanofibers," Materials Science and Engineering A, vol. 459, no. 1-2, pp. 390-396, 2007.

[9] J. S. Rodrigues, N. S. Santos-Magalhães, L. C. B. B. Coelho, P. Couvreur, G. Ponchel, and R. Gref, "Novel core(polyester)shell(polysaccharide) nanoparticles: protein loading and surface modification with lectins," Journal of Controlled Release, vol. 92, no. 1-2, pp. 103-112, 2003.

[10] X. Y. Yang, X. Y. Zhang, Z. F. Liu, Y. F. Ma, Y. Huang, and Y. S. Chen, "High-efficiency loading and controlled release of doxorubicin hydrochloride on graphene oxide," Journal of Physical Chemistry C, vol. 112, no. 45, pp. 17554-17558, 2008.

[11] O. C. Farokhzad and R. Langer, "Impact of nanotechnology on drug delivery," ACS Nano, vol. 3, no. 1, pp. 16-20, 2009.

[12] K. Yang, L. Z. Feng, X. Z. Shi, and Z. Liu, "Nano-graphene in biomedicine: theranostic applications," Chemical Society Reviews, vol. 42, no. 2, pp. 530-547, 2013.

[13] A. H. Stegh, "Toward personalized cancer nanomedicine: past, present, and future," Integrative Biology, vol. 5, no. 1, pp. 48-65, 2013.

[14] Y. J. Lu, H. W. Yang, S. C. Hung et al., "Improving thermal stability and efficacy of BCNU in treating glioma cells using PAA-functionalized graphene oxide," International Journal of Nanomedicine, vol. 7, no. 1, pp. 1737-1747, 2012.

[15] A. K. Geim and K. S. Novoselov, "The rise of graphene," Nature Materials, vol. 6, no. 3, pp. 183-191, 2007.

[16] M. J. Allen, V. C. Tung, and R. B. Kaner, "Honeycomb carbon: a review of graphene," Chemical Reviews, vol. 110, no. 1, pp. 132$145,2010$.

[17] G. Eda and M. Chhowalla, "Chemically derived graphene oxide: towards large-area thin-film electronics and optoelectronics," Advanced Materials, vol. 22, no. 22, pp. 2392-2415, 2010.

[18] K. Wang, J. Ruan, H. Song et al., "Biocompatibility of graphene oxide," Nanoscale Research Letters, vol. 6, no. 1, article 8, 2011. 
[19] G. Gollavelli and Y. C. Ling, "Multi-functional graphene as an in vitro and in vivo imaging probe," Biomaterials, vol. 33 , no. 8 , pp. 2532-2545, 2012.

[20] D. Bitounis, H. A. Boucetta, B. H. Hong, D. H. Min, and K. Kostarelos, "Prospects and challenges of graphene in biomedical applications," Advanced Materials, vol. 25, no. 16, pp. 22582268, 2013.

[21] N. G. Sahoo, H. Q. Bao, Y. Z. Pan et al., "Functionalized carbon nanomaterials as nanocarriers for loading and delivery of a poorly water-soluble anticancer drug: a comparative study," Chemical Communications, vol. 47, no. 18, pp. 5235-5237, 2011.

[22] L. Z. Feng and Z. Liu, "Graphene in biomedicine: opportunities and challenges," Nanomedicine, vol. 6, no. 2, pp. 317-324, 2011.

[23] X. X. Ma, H. Q. Tao, K. Yang et al., "A functionalized graphene oxide-iron oxide nanocomposite for magnetically targeted drug delivery, photothermal therapy, and magnetic resonance imaging," Nano Research, vol. 5, no. 3, pp. 199-212, 2012.

[24] X. Sun, Z. Liu, K. Welsher et al., "Nano-graphene oxide for cellular imaging and drug delivery," Nano Research, vol. 1, no. 3, pp. 203-212, 2008.

[25] K. Liu, J. J. Zhang, F. F. Cheng, T. T. Zheng, C. Wang, and J. J. Zhu, "Green and facile synthesis of highly biocompatible graphene nanosheets and its application for cellular imaging and drug delivery," Journal of Materials Chemistry, vol. 21, no. 32, pp. 12034-12040, 2011.

[26] H. Hu, J. Yu, Y. Li, J. Zhao, and H. Dong, "Engineering of a novel pluronic F127/graphene nanohybrid for $\mathrm{pH}$ responsive drug delivery," Journal of Biomedical Materials Research A, vol. 100, no. 1, pp. 141-148, 2012.

[27] H. Wen, C. Dong, H. Dong et al., "Engineered redox-responsive PEG detachment mechanism in PEGylated nano-graphene oxide for intracellular drug delivery," Small, vol. 8, no. 5, pp. 760-769, 2012.

[28] H. Bao, Y. Pan, Y. Ping et al., "Chitosan-functionalized graphene oxide as a nanocarrier for drug and gene delivery," Small, vol. 7, no. 11, pp. 1569-1578, 2011.

[29] Y. Pan, H. Bao, N. G. Sahoo, T. Wu, and L. Li, "Water-soluble poly(N-isopropylacrylamide)-graphene sheets synthesized via click chemistry for drug delivery," Advanced Functional Materials, vol. 21, no. 14, pp. 2754-2763, 2011.

[30] Z. Liu, J. T. Robinson, X. M. Sun, and H. J. Dai, "PEGylated nanographene oxide for delivery of water-insoluble cancer drugs," Journal of the American Chemical Society, vol. 130, no. 33, pp. 10876-10877, 2008.

[31] M. Kakran, N. G. Sahoo, H. Bao, Y. Pan, and L. Li, "Functionalized graphene oxide as nanocarrier for loading and delivery of ellagicacid," Current Medicinal Chemistry, vol. 18, no. 29, pp. 4503-4512, 2011.

[32] X. T. Zheng and C. M. Li, "Restoring basal planes of graphene oxides for highly efficient loading and delivery of $\beta$-lapachone," Molecular Pharmaceutics, vol. 9, no. 3, pp. 615-621, 2012.

[33] M. D. Stoller, S. Park, Y. W. Zhu, J. H. An, and R. S. Ruoff, "Graphene-based ultracapacitors," Nano Letters, vol. 8, no. 10, pp. 3498-3502, 2008.

[34] J. M. Bak and H. Lee, "pH-tunable aqueous dispersion of graphene nanocomposites functionalized with poly(acrylic acid) brushes," Polymer, vol. 53, no. 22, pp. 4955-4960, 2012.

[35] B. S. Shen, J. J. Ding, X. B. Yan, W. J. Feng, J. Li, and Q. J. Xue, "Influence of different buffer gases on synthesis of few-layered graphene by arc discharge method," Applied Surface Science, vol. 258, no. 10, pp. 4523-4531, 2012.
[36] K. S. Subrahmanyam, L. S. Panchakarla, A. Govindaraj, and C. N. R. Rao, "Simple method of preparing graphene flakes by an arc-discharge method," Journal of Physical Chemistry C, vol. 113, no. 11, pp. 4257-4259, 2009.

[37] Z. S. Wu, W. C. Ren, L. B. Gao et al., "Synthesis of graphene sheets with high electrical conductivity and good thermal stability by hydrogen arc discharge exfoliation," ACS Nano, vol. 3, no. 2, pp. 411-417, 2009.

[38] N. Li, Z. Wang, K. Zhao, Z. J. Shi, Z. N. Gu, and S. K. Xu, "Large scale synthesis of $\mathrm{N}$-doped multi-layered graphene sheets by simple arc-discharge method," Carbon, vol. 48, no. 1, pp. 255259, 2010.

[39] Y. P. Wu, B. Wang, Y. F. Ma et al., "Efficient and largescale synthesis of few-layered graphene using an arc-discharge method and conductivity studies of the resulting films," Nano Research, vol. 3, no. 9, pp. 661-669, 2010.

[40] J. Li, J. T. Chen, B. S. Shen, X. B. Yan, and Q. J. Xue, “Temperature dependence of the field emission from the few-layer graphene film," Applied Physics Letters, vol. 99, no. 16, Article ID 163103, 3 pages, 2011.

[41] J. An, Y. Q. Gou, C. X. Yang, F. D. Hu, and C. M. Wang, "Synthesis of a biocompatible gelatin functionalized graphene nanosheets and its application for drug delivery," Materials Science and Engineering C, vol. 33, no. 5, pp. 2827-2837, 2013.

[42] Z. X. Tai, H. B. Ma, B. Liu, X. B. Yan, and Q. J. Xue, "Facile synthesis of Ag/GNS-g-PAA nanohybrids for antimicrobial applications," Colloids and Surfaces B, vol. 89, no. 1, pp. 147-151, 2012.

[43] Z. Guo, F. Du, D. M. Ren et al., "Covalently porphyrinfunctionalized single-walled carbon nanotubes: a novel photoactive and optical limiting donor-acceptor nanohybrid," Journal of Materials Chemistry, vol. 16, no. 29, pp. 3021-3030, 2006.

[44] K. F. Ilett, R. T. T. Ong, K. T. Batty, and J. D. Taylor, "Effect of urine $\mathrm{pH}$ on the stability of doxorubicin and its recovery from bladder instillations," The British Journal of Urology, vol. 65, no. 5, pp. 478-482, 1990.

[45] M. J. Wood, W. J. Irwin, and D. K. Scott, "Photodegradation of doxorubicin, daunorubicin and epirubicin measured by high-performance liquid chromatography," Journal of Clinical Pharmacy and Therapeutics, vol. 15, no. 4, pp. 291-300, 1990. 

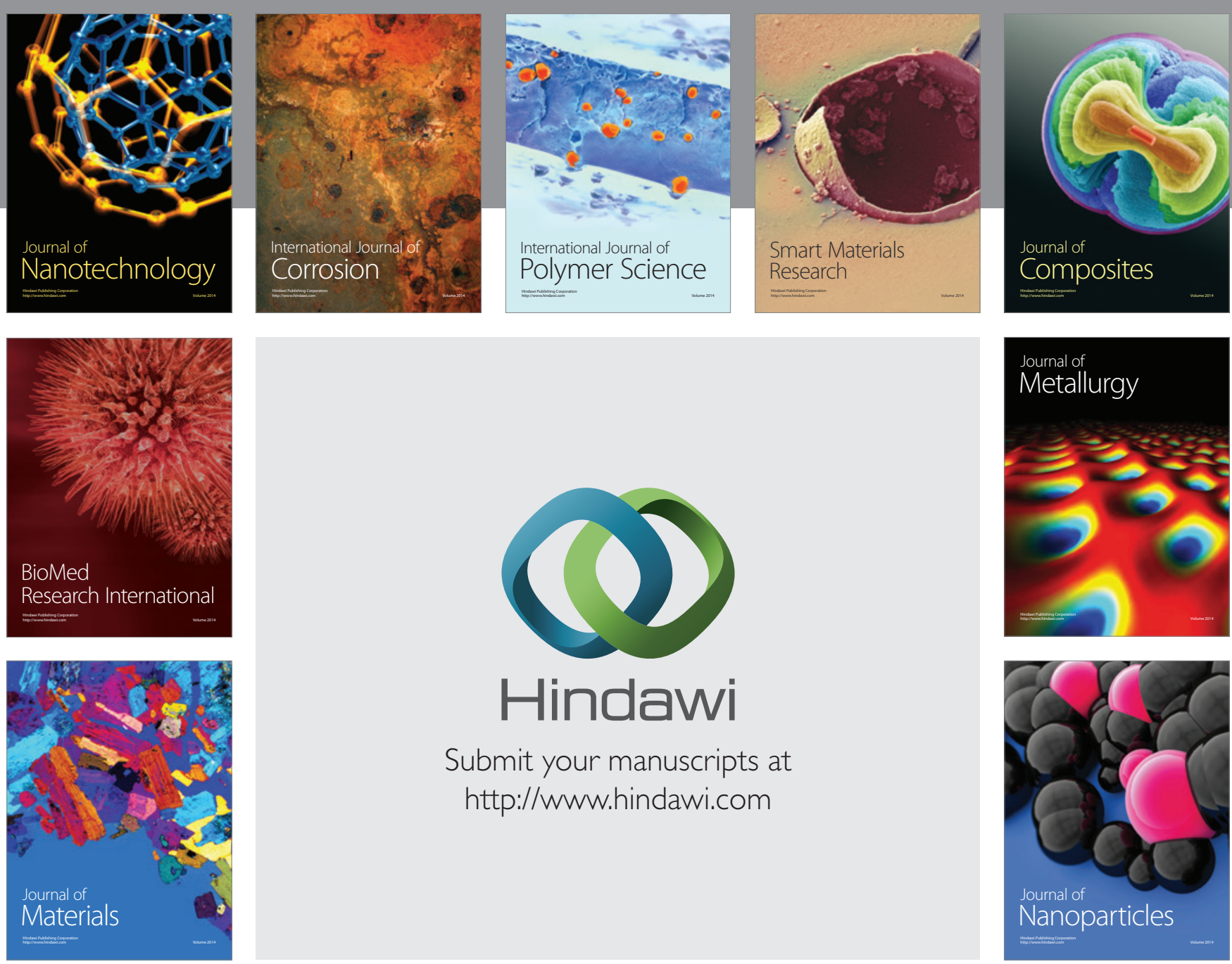

Submit your manuscripts at http://www.hindawi.com
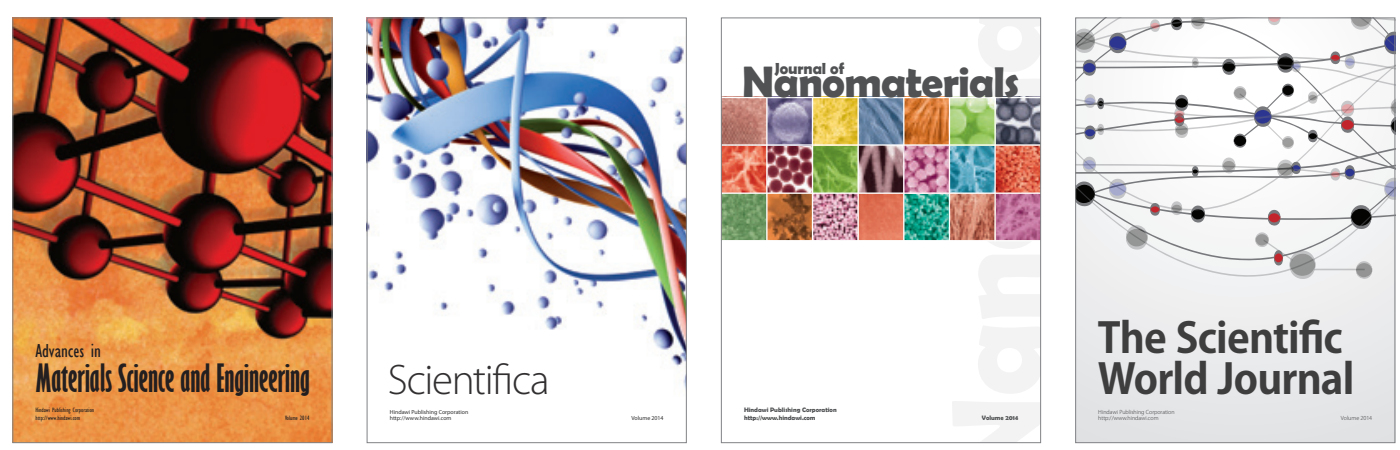

\section{The Scientific World Journal}
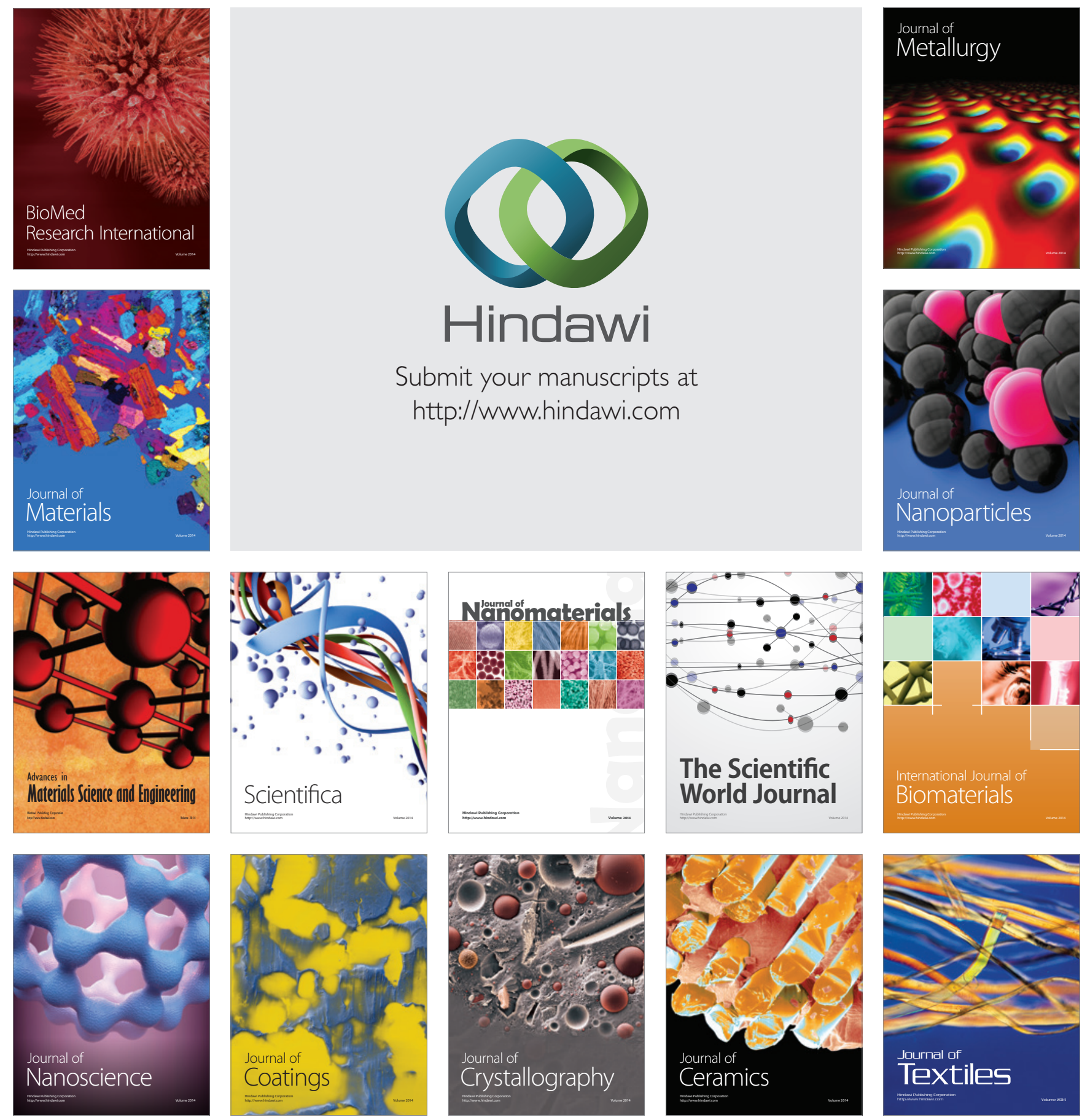